\title{
AVALIAÇÃO DA ATIVIDADE FÍSICA DE ESCOLARES COM UM QUESTIONÁRIO VIA INTERNET
}

\author{
ASSESSMENT OF PHYSICAL ACTIVITY IN SCHOOLCHILDREN USING A WEB-BASED QUESTIONNAIRE
}

Artigo Original

EVALUACIÓN DE LA ACTIVIDAD FÍSICA DE ESCOLARES CON UN CUESTIONARIO BASADO EN LA INTERNET

Artigo Original
Original ArticLe

Artículo Original

Gilmar Mercês de Jesus ${ }^{1}$

(Educador Físico)

Maria Alice Altenburg de Assis ${ }^{1,2}$

(Nutricionista)

Emil Kupek ${ }^{3}$ (Epidemiologista)

Lizziane Andrade Dias ${ }^{4}$

(Educador Físico)

\begin{abstract}
1. Programa de Pós-Graduação em Educação Física, Universidade Federal de Santa Catarina,

Florianópolis, SC, Brasil.

2. Programa de Pós-Graduação em Nutrição, Universidade Federal de Santa Catarina, Florianópolis, SC, Brasil.

3. Programa de Pós-Graduação em Saúde Coletiva, Universidade Federal de Santa Catarina, Florianópolis, SC, Brasil. 4. Universidade Estadual de Feira de Santana, Feira de Santana, BA, Brasil.
\end{abstract}

\section{Correspondência:}

Universidade Estadual de Feira de Santana

Departamento de Saúde, MT65,

Núcleo de Estudos e Pesquisas em Atividade Física e Saúde (NEPAFIS), Avenida Transnordestina, S/N, Bairro Novo Horizonte, Feira de Santana, BA, Brasil. 44036-900. gilmarmerces@gmail.com

\section{RESUMO}

Introdução: A atividade física é um comportamento que se estabelece na infância, tende a se manter na vida adulta e influencia a regulação do balanço energético, do peso e da adiposidade corporal, prevenindo a obesidade. Por isso, criar e validar métodos de avaliação e monitoramento da atividade física de crianças e adolescentes no nível populacional é necessário para formular políticas públicas. Objetivo: Avaliar validade e reprodutibilidade de comportamentos sedentários e atividades físicas autorrelatadas no questionário Consumo Alimentar e Atividade Física de Escolares (Web-CAAFE), um instrumento computacional baseado na internet. Métodos: Escolares do $2^{\circ}$ ao $5^{\circ}$ ano $(n=390)$ foram observados durante o horário do recreio e preencheram o questionário no dia seguinte. Uma subamostra $(n=94)$ preencheu o Web-CAAFE duas vezes com três horas de intervalo. O custo energético, como equivalentes metabólicos (MET), foi calculado para cada atividade física e somado ao nível do indivíduo. O MET individual de atividades físicas e o total de comportamentos sedentários autorrelatados foram os principais desfechos analisados separadamente, utilizando regressão de Poisson e regressão de Poisson hierárquica (aluno no primeiro nível e a repetição dos métodos no segundo nível). Os resultados foram apresentados como razões de incidências (RI). Os resíduos foram analisados graficamente por sexo e idade. Resultados: O Web-CAAFE superestimou o total de comportamentos sedentários ( $R I=2,90$, IC 95\%: 2,05-4,10), principalmente entre as garotas mais jovens (7-10 anos), enquanto as mais velhas (11-15 anos) tenderam à subestimação. A superestimação nos MET de atividades físicas foi menor e não estatisticamente significante. Não houve diferença estatisticamente significante no total de comportamentos sedentários ou MET de atividades físicas entre o primeiro e o segundo autorrelato. Conclusão: O Web-CAAFE mostrou boa confiabilidade e maior precisão quando aplicado à avaliação de MET de atividades físicas dos estudantes mais velhos.

Descritores: validade dos testes; reprodutibilidade dos resultados; atividade motora; estudantes.

\section{ABSTRACT}

Introduction: Physical activity is a behavior that is established during childhood and tends to be maintained in adulthood, influencing the regulation of the energy balance, weight and body fat, and thereby preventing obesity. Therefore, creating and validating methods to assess and monitor this activity in children and adolescents across the whole population is very important for guiding public health policies. Objective: To assess the validity and reproducibility of self-reported sedentary behaviors and physical activity, through the web-based Questionnaire Consumo Alimentar e Atividade Fisica de Escolares (Web-CAAFE, Food Intake and Physical Activity of School Children). Methods: Students in the $2^{\text {nd }}$ to $5^{\text {th }}$ grades $(n=390)$ were directly observed during break times, and were asked to fill out the Web-CAAFE questionnaire the day after. A subsample $(n=94)$ completed the Web-CAAFE twice, with an interval of three hours between each time. The energy cost was calculated for each physical activity as metabolic equivalents (MET) and added to the individual level. The individual MET for the physical activities and total self-reported sedentary behaviors were the main outcomes, analyzed separately by Poisson regression and hierarchical Poisson regression (student as the first level and repetition of the methods in the second level). The results were presented as Incidence Ratios (IR). The residuals were analyzed graphically by gender and age. Results: The Web-CAAFE overestimated the total sedentary behaviors (IR=2.90, CI 95\%: 2.05-4.10), especially among younger girls (7-10 years old), while for the older girls (11-15 years old), it tended to underestimation. The overestimation of MET for physical activity was lower, and was not statistically significant. No statistically significant difference was found between the first and second self-reported questionnaires, either for total sedentary behaviors or MET for physical activity. Conclusion: The Web-CAAFE showed good reliability and high precision when applied to the assessment of MET for physical activity in the older students.

Keywords: validity of tests; reproducibility of results; motor activity; students.

\section{RESUMEN}

Introducción: La actividad física es un comportamiento que se establece en la infancia, tiende a permanecer en la edad adulta y que influye en la regulación del balance energético, del peso y de la grasa corporal, previniendo la obesidad. Por lo tanto, crear y validar métodos de evaluación y monitoreo de la actividad física de los niños y adolescentes a nivel de la población es necesario para formulación de políticas públicas. Objetivo: Evaluar la validez y la reproducibilidad de los comportamientos sedentarios y las actividades físicas auto-informadas en el cuestionario Consumo Alimentario y Actividad Física de los Escolares (Web-CAAFE), herramienta computacional basada en Internet. Métodos: Se observaron 
los alumnos del $2^{\circ}$ al $5^{\circ}$ año $(n=390)$ durante el tiempo de recreo que completaron el cuestionario al día siguiente. Una submuestra ( $n=94)$ completó el Web-CAAFE dos veces con intervalos de tres horas. El costo de energético, en equivalentes metabólicos (MET), se calculó para cada actividad física y fue añadido al nivel individual. El MET individual de actividades físicas y el total de los comportamientos sedentarios auto-informados fueron los principales resultados analizados por separado, utilizando regresión de Poisson y Poisson jerárquica (estudiante en el primer nivel y la repetición de los métodos en el segundo nivel). Los resultados se presentaron como tasa de incidencia (TI). Los residuos se analizaron gráficamente por sexo y edad. Resultados: El Web-CAAFE ha sobreestimado los comportamientos sedentarios ( $T$ I $=2,90,1 C$ del 95\%: 2,5-4,10), especialmente entre las niñas más jóvenes (7-10 años), mientras que las mayores (11-15 años) tendían a la subestimación. La sobreestimación de los MET de las actividades físicas era menory no fue estadísticamente significativa. No hubo diferencia estadísticamente significativa en el total de comportamientos sedentarios o el MET de actividades fisicas entre el primery el segundo auto-informe. Conclusión: EI Web-CAAFE mostró una buena fiabilidad y mayor precisión cuando se aplica a la evaluación del MET de actividades físicas de los estudiantes de mayor edad.

Descriptores: validez de las pruebas; reproducibilidad de resultados; actividad motora; estudiantes.

\section{INTRODUÇÃO}

A obesidade infantil é um importante problema de saúde pública em todo o mundo, por sua alta prevalência e associação com doenças crônicas ${ }^{1}$.

A atividade física desempenha importante papel na etiologia da obesidade, influenciando o balanço energético ${ }^{2}$, o peso ${ }^{3}$ e a adiposidade corporal $^{4}$, e é um comportamento que se estabelece durante a infância, tendendo a se manter durante a vida adulta ${ }^{5}$. Assim, é necessário dispor de métodos precisos e confiáveis para sua avaliação e monitoramento em âmbito populacional. Isto tem importante implicação para as pesquisas epidemiológicas sobre tendências e associações com doenças, e para a formulação de estratégias de enfrentamento da obesidade e de avaliação do impacto de intervenções em políticas de saúde ${ }^{6,7}$.

Tem crescido o uso de questionários computacionais em pesquisas epidemiológicas que possibilitam obter informações rapidamente, reduzem custos, garantem rápido acesso aos dados, e eliminam a fase de digitação, evitando muitos vieses ${ }^{8}$. Questionários computacionais são alternativas promissoras para pesquisas populacionais sobre a atividade física9, ${ }^{9}$, pois são mais atrativos para os respondentes e apresentam grande potencial de expansão, considerando o amplo acesso da população às novas tecnologias da informação, sobretudo computadores e internet.

No Brasil, o questionário Consumo Alimentar e Atividade Física de Escolares (Web-CAAFE) ${ }^{11,12}$, um instrumento computacional baseado na internet, foi desenvolvido para um sistema de monitoramento de abrangência nacional, possibilitando avaliar estado nutricional, consumo alimentar e atividades físicas e comportamentos sedentários de escolares de sete a 10 anos de idade.

O Web-CAAFE apresentou alta sensibilidade e especificidade na avaliação da atividade física de escolares do ensino fundamental público da cidade de Florianópolis-SC, Brasil, quando comparado à observação direta ${ }^{13}$. Porém, novas pesquisas são necessárias com a finalidade de comparar dados com outras cidades brasileiras e para o refinamento do instrumento.

O presente estudo determinou reprodutibilidade teste-reteste e validade de critério de atividades físicas e comportamentos sedentários autorrelatados no Web-CAAFE por escolares do ensino fundamental de uma cidade de grande porte da região Nordeste do Brasil.

\section{MÉTODOS}

A amostra foi de conveniência e incluiu estudantes do segundo ao quinto ano do ensino fundamental de uma escola pública de Feira de Santana-BA, uma cidade de grande porte do Nordeste do Brasil. A escola selecionada atendeu ao protocolo de pesquisa que previa a realização do estudo numa escola pública de ensino fundamental, com sala informatizada e acesso à internet, interesse do diretor e dos professores em colaborar com a pesquisa e oferta de alimentação escolar. Igualmente às demais escolas de primeiro ao quinto ano da cidade, a escola selecionada não oferecia aulas de Educação Física. Dessa forma, as atividades físicas praticadas pelos estudantes no período escolar ocorriam prioritariamente no horário do recreio.

Foram incluídos no estudo os estudantes com frequência na ocasião da coleta de dados $(n=453)$. O estudo foi aprovado pelo Comitê de Ética em Pesquisa a Universidade Estadual de Feira de Santana (CAAE: 19499913.3.0000.0053). Os participantes receberam autorização por escrito dos pais e assinaram o Termo de Consentimento Livre e Esclarecido.

O Web-CAAFE é um software para o recordatório do dia anterior, dispondo de um avatar animado que auxilia o preenchimento ${ }^{13}$. $\mathrm{O}$ instrumento contém um formulário de registro, uma seção sobre consumo alimentar e uma sobre atividades físicas e comportamentos sedentários, incluindo formas de deslocamento para a escola. As atividades físicas e comportamentos sedentários são autorrelatados em três períodos do dia (manhã, tarde e noite), selecionando-se figuras.

No estudo de validade de critério adotou-se como padrão de referência a observação direta dos alunos durante o recreio, de forma similar ao empregado em outras publicações ${ }^{13,14}$. No dia seguinte à observação os participantes preencheram o Web-CAAFE.

A equipe de observadores incluiu professores de Educação Física, Nutricionistas e estudantes de Nutrição, Educação Física e Biomedicina, previamente treinados. Os observadores foram posicionados em diferentes setores e anotaram as atividades prioritárias dos estudantes que estavam identificados com pulseiras coloridas e crachás com numeração codificada. As anotações eram comparadas ao final do recreio e então o registro oficial de observação era preenchido. Foram consideradas prioritárias as atividades estruturadas e aquelas anotadas por mais de um observador para o mesmo estudante. Discordâncias eram resolvidas por diálogo. Esta etapa foi precedida pela simulação da coleta de dados, para reduzir a reatividade dos estudantes aos pesquisadores e aos procedimentos.

No estudo da reprodutibilidade teste-reteste uma subamostra foi sorteada entre os concluintes da etapa da validade, repetindo-se todo o procedimento com a adição de uma segunda aplicação do Web-CAAFE três horas após a primeira. Assim, os dados analisados compreenderam duas observações diretas e três autorrelatos para cada criança participante de ambas etapas do estudo.

Os dados foram analisados com o software STATA 12.0, assumindo-se 
nível de significância estatística de $p \leq 0,05$ para testes de hipóteses e intervalos de confiança de 95\% para inferência estatística. A análise incluiu estatística descritiva e Regressão de Poisson para avaliar validade e reprodutibilidade, apresentando os resultados como razões de incidências (RI).

Valores em equivalentes metabólicos (MET) foram atribuídos às atividades do Web-CAAFE, utilizando o Compendium of Energy Expenditures for Youth ${ }^{15}$. O custo energético, como equivalentes metabólicos (MET), foi calculado para cada atividade física e somado no nível de indivíduo. Assim, MET de atividades físicas e o número de comportamentos sedentários autorrelatados foram os desfechos analisados com a Regressão de Poisson hierárquica (multinível), sendo o aluno o primeiro nível e a repetição dos métodos o segundo.

Os modelos de regressão foram ajustados após a exclusão de valores extremos (outliers) com base nos valores máximos observados e com base na distribuição de Poisson, utilizando como critério de exclusão os valores que ultrapassaram as médias dos desfechos autorrelatados mais três vezes o desvio padrão. Este último critério teve em vista a aplicação rotineira do Web-CAAFE como sistema de monitoramento, supondo a indisponibilidade de dados observados diretamente para avaliar a fidedignidade das respostas autorrelatadas.

A supradispersão do modelo foi investigada graficamente (detaIhes não apresentados), relacionando os resíduos com os desfechos, assim como via regressão binomial negativa que assumiu dispersão proporcional à média do desfecho, efeitos fixos para tipo de instrumento e para repetição, e efeitos randômicos entre os indivíduos. O modelo final foi selecionado pela comparação dos desvios entre os modelos analisados, apreciando graus de liberdade. Analisou-se graficamente a magnitude e a distribuição dos resíduos, com relação a sexo e idade.

\section{RESULTADOS}

Dos escolares incluídos, 91,8\% aceitaram participar e obtiveram autorização por escrito dos pais, 3,8\% não retornaram o consentimento escrito, 4\% não obtiveram consentimento dos pais, e dois meninos do quarto e do quinto ano $(0,4 \%)$, respectivamente, recusaram participar. Concluíram o estudo 390 estudantes [Média de idade (anos) \pm Desvio Padrão = 9,53 $\pm 1,53$ anos] dos quais, 94 participaram também da etapa da reprodutibilidade $(9,41 \pm 1,44$ anos).

A Tabela 1 apresenta as características da amostra. A faixa etária dos estudantes na escola pesquisada era de sete a 15 anos.

A Tabela 2 apresenta as frequências das atividades físicas e comportamentos sedentários diretamente observados e autorrelatados. Os padrões de distribuição dos itens foram similares: as frequências decresceram igualmente, com exceção da atividade "ouvir música".

Foram detectados 18 outliers (4,6\%) que modificaram significativamente os valores médios dos desfechos analisados (variação de 20\% a 37\%), justificando sua exclusão para as análises de regressão (Tabela 3).

Tabela 1. Características dos participantes.

\begin{tabular}{c|c|c}
\hline \multirow{2}{*}{ Característica } & Validade $(\mathbf{n}=\mathbf{3 9 0})$ & Reprodutibilidade $(\mathbf{n}=\mathbf{9 4})$ \\
\cline { 2 - 3 } & $\mathbf{( \% )}$ & $\mathbf{( \% )}$ \\
\hline Sexo & & 50,0 \\
\hline Masculino & 49,7 & 50,0 \\
\hline Feminino & 50,3 & \\
\hline Idade (anos) & & 17,0 \\
\hline 7 & 18,2 & 20,2 \\
\hline 8 & 18,2 & 29,8 \\
\hline 9 & 24,9 & 21,3 \\
\hline 10 & 24,9 & 7,4 \\
\hline 11 & 8,2 & 4,3 \\
\hline $12-15$ & 5,6 & \\
\hline
\end{tabular}

Em comparação com os dados observados, houve superestimação dos relatos de comportamentos sedentários e de atividades físicas no Web-CAAFE, quando se analisou os itens individualmente, excetuando a atividade "pega-pega", subestimada no autorrelato (Tabela 2). Os maiores vieses entre Web-CAAFE e padrão de referência ocorreram nas atividades que apresentaram menor frequência de autorrelato.

A frequência do total de comportamentos sedentários foi quase três vezes maior no autorrelato $\left(R \mid=2,90, I C_{95 \%}: 2,05-4,10\right)$. Conquanto tenha sido notada superestimação de $18 \%$ para MET de atividades físicas autorrelatadas, tal resultado não foi estatisticamente significante $(\mathrm{Rl}=1,18 p=0,079)$ (Tabela 4).

A variabilidade inter-sujeitos para o total de comportamentos sedentários autorrelatados foi alta, oscilando de 6\% a 179\%. Esse efeito foi menor e não estatisticamente significante para MET de atividades físicas (10\%) (Tabela 4).

A magnitude dos resíduos indicou superestimação do total de comportamentos sedentários de meninas mais jovens (7-10 anos) e subestimação das mais velhas (11-15 anos) (Figura 1). Resultado similar ocorreu para meninos, porém esta tendência não apresentou a mesma clareza. Houve superestimação de MET de atividades físicas entre meninos, com diminuição da tendência com a idade, enquanto houve subestimação entre meninas, com semelhante tendência em função da idade (Figura 2).

Apenas as atividades, "amarelinha" e "brincar no celular" foram mais superestimadas no segundo autorrelato em comparação com o primeiro. Não houve diferença estatisticamente significante para os demais itens do questionário no segundo autorrelato em comparação com o primeiro, quando analisados individualmente (Tabela 2).

Também não houve diferença estatisticamente significante no total de comportamentos sedentários nem em MET de atividades físicas na segunda aplicação do Web-CAAFE em comparação com a primeira (Tabela 4).

\section{DISCUSSÃO}

Comportamentos sedentários foram significativamente superestimados no Web-CAAFE quando comparados à observação direta, principalmente entre os estudantes mais jovens. A superestimação foi menor e não estatisticamente significativa para MET de atividades físicas, ocorrendo principalmente entre meninos mais jovens, com tendência à diminuição com a idade, enquanto houve subestimação entre meninas mais jovens, com similar tendência relacionada à idade.

A ausência de diferenças tanto no total de comportamentos sedentários quanto em MET de atividades físicas autorrelatadas no intervalo de três horas entre a segunda e a primeira aplicação do Web-CAAFE sugere adequada confiabilidade do instrumento na avaliação da amostra pesquisada.

Comparações com outros estudos são limitadas pelas diferenças no padrão de referência e na análise dos dados. A medida da atividade física com o uso de pedômetros e acelerômetros tem sido o padrão de referência mais utilizado em estudos de validade envolvendo questionários computacionais para crianças e adolescentes ${ }^{9}$. Contudo, no presente estudo o padrão de referência para a validade de critério, foi a observação direta.

A observação direta também é um método adequado de avaliação da atividade física ${ }^{16}$ e mostrou vantagens neste estudo, pois independe da memória dos participantes e dos erros relacionados com as medidas (Web-CAAFE e padrão de referência), e possibilitou obter informações do tipo de atividade física, sendo, portanto, condizente com o Web-CAAFE.

De modo geral, instrumentos computacionais de avaliação da atividade física de crianças e adolescentes apresentam correlações fracas a moderadas com acelerômetros, mas boa confiabilidade ${ }^{17-25}$. 
Tabela 2. Validade e reprodutibilidade dos comportamentos sedentários e atividades físicas autorrelatados no Web-CAAFE.

\begin{tabular}{|c|c|c|c|c|c|c|c|c|}
\hline \multirow{3}{*}{ Comportamentos sedentários } & \multicolumn{4}{|c|}{ Validade } & \multicolumn{4}{|c|}{ Reprodutibilidade } \\
\hline & \multicolumn{2}{|r|}{$\%$} & \multirow{2}{*}{$\begin{array}{c}\text { Viés } \\
(\text { IC 95\% })^{\dagger}\end{array}$} & \multirow{2}{*}{ P-valor } & \multicolumn{2}{|c|}{$\%$} & \multirow{2}{*}{$\begin{array}{c}\text { RI } \\
(\text { IC } 95 \%)^{\ddagger} \\
\end{array}$} & \multirow{2}{*}{ P-valor } \\
\hline & $\mathrm{OD}^{*}$ & Web-CAAFE & & & Teste & Re-teste & & \\
\hline \multirow{2}{*}{ Estudar/Ler/ Escrever/Desenhar/Pintar } & \multirow{2}{*}{11,4} & \multirow{2}{*}{22,6} & 1,96 & \multirow{2}{*}{0,000} & \multirow{2}{*}{22,7} & & 1,13 & \\
\hline & & & $(1,44-2,71)$ & & & 22,3 & $(0,84-1,55)$ & 0,412 \\
\hline Assistir TV & & & 58,99 & & & & 0,7 & \\
\hline Assistir IV & 0,2 & 11,8 & $(8,14-427,70)$ & 0,000 & 12,9 & 9,2 & $(0,36-1,36)$ & 0,298 \\
\hline Usar o computador & - & 11,2 & - & - & 11,9 & 9,2 & - & - \\
\hline Video game & - & 6,9 & - & - & - & 6,9 & - & - \\
\hline Brincar no colular & 23 & 138 & 3,82 & ( & 0.5 & 246 & 2,32 & ( \\
\hline DIIILar tio celutal & 3,3 & 13,0 & $(2,31-6,30)$ & 0,000 & प, & 24,0 & $(1,52-3,55)$ & 0,000 \\
\hline Atividades físicas & & & & & & & & \\
\hline & & & 0,52 & & & & 0,87 & \\
\hline Pega-pega & 58,1 & 30 & $(0,45-0,61)$ & 0,000 & 32,2 & 24,6 & $(0,71-1,05)$ & 0,165 \\
\hline Futebol & 11 & 127 & 1,17 & 0269 & 132 & 115 & 0,91 & 0722 \\
\hline rutenol & 11 & 12,1 & $(0,88-1,55)$ & 0,209 & 13,2 & 11,5 & $(0,54-1,52)$ & $0,12 \angle$ \\
\hline Fsconde-esconde & 31 & 66 & 2,2 & 0.001 & 71 & 54 & 0,63 & 0.304 \\
\hline escumae-escomide & 3,1 & 0,0 & $(1,35-3,58)$ & 0,001 & 7,1 & 0,4 & $(0,26-1,52)$ & 0,504 \\
\hline & & & 2,36 & & & & 1,14 & \\
\hline Uançar & 2,5 & 5,9 & $(1,32-4,23)$ & 0,004 & 4,9 & 8,5 & $(0,58-2,23)$ & $0, / 11$ \\
\hline Brincar com honeca/boneco & & & 3,59 & & & & 1,04 & \\
\hline Brincar com doneca/doneco & 1 & 3,1 & $(1,31-9,86)$ & 0,013 & 3,1 & 3,8 & $(0,38-2,89)$ & 0,935 \\
\hline & & & 5,15 & & & & 1,35 & \\
\hline Pular corda & 0,6 & 3,3 & $(1,60-16,53)$ & 0,006 & 2,8 & 4,6 & $(0,54-3,36)$ & 0,513 \\
\hline Amarelinha & & & 8,16 & & & 77 & 3,96 & \\
\hline Amaremnnd & 0,4 & 3,9 & $(1,92-34,71)$ & 0,004 & 2,5 & 7, I & $(1,55-10,10)$ & 0,004 \\
\hline & & & 18,1 & & & & 0,99 & \\
\hline ouvir musica & 0,2 & $3 /, 3$ & $(2,41-135,2)$ & 0,005 & 3,1 & 3,8 & $(0,36-2,69)$ & 0,982 \\
\hline Jogo com bola & - & 31 & $2=$ & - & 25 & 46 & 1,88 & 0236 \\
\hline Jogo com Dold & - & 3,1 & - & - & 2,0 & 4,0 & $(0,66-5,35)$ & 0,230 \\
\hline & & & & & & & 0,84 & \\
\hline Andar de Dicicleta & - & 4,4 & - & - & 4,6 & 3,8 & $(0,31-2,25)$ & 0,723 \\
\hline Lavar louca & - & 4,6 & - & - & 4,6 & 46 & 1 & 0,995 \\
\hline Lavdl IUUÇa & - & 4,0 & - & - & 4,0 & 4,0 & $(0,36-2,82)$ & 0,990 \\
\hline & & & & & & & 1,09 & \\
\hline Karate/capoelra & - & 5 & - & - & 4,9 & 5,4 & $(0,46-2,61)$ & 0,834 \\
\hline Brincar na piscina & - & 3,9 & - & - & 4,3 & 3,1 & - & - \\
\hline Varrer & & 35 & - & & & & 0,84 & \\
\hline varrer & - & 3,5 & - & - & 3,6 & 3,1 & $(0,27-2,54)$ & $0, / 52$ \\
\hline Bolinha de aude & - & 35 & _- & _- & 37 & 31 & 0,84 & 0752 \\
\hline & & & - & - & 3,1 & 3,1 & $(0,28-2,54)$ & 0,152 \\
\hline Brincar com o cachorro & _- & 37 & _ & _- & & 38 & 1,04 & 0.941 \\
\hline Brincar com o cachorro & - & 3,1 & - & - & 3,1 & 3,8 & $(0,33-3,34)$ & 0,941 \\
\hline Ginástica & - & 1,8 & - & - & 1,8 & 1,5 & 0,84 & 0,825 \\
\hline Glindicd & - & 1,0 & - & - & 1,8 & 1,5 & $(0,17-4,10)$ & 0,825 \\
\hline Balé & - & 2,4 & - & - & 2,5 & 2,3 & - & - \\
\hline Pipa & - & 2,9 & - & - & 2,5 & 3,8 & - & - \\
\hline Carrinho & - & 1,8 & - & - & 18 & & 0,83 & \\
\hline Carrinno & - & 1,8 & - & - & 1,8 & 1,5 & $(0,17-4,10)$ & 0,825 \\
\hline Elástico & - & 1,5 & - & - & 1,5 & 1,5 & - & - \\
\hline Brincar no parque & - & 1,3 & - & - & 15 & 08 & 1 & 1.000 \\
\hline Dilicar ho parque & - & 1,3 & - & - & 1,5 & 0,8 & $(0,55-18,17)$ & 1,000 \\
\hline Patins/Skate/Patinete & - & 13 & - & - & 12 & 15 & 1,25 & 0,793 \\
\hline Patins/skate/Patinete & - & 1,3 & - & - & 1,2 & 1,5 & $(0,23-6,79)$ & 0,193 \\
\hline Jogos de tabuleiro & - & 88 & - & - & 06 & 15 & 2,51 & 0354 \\
\hline & - & 0,0 & - & - & 0,0 & 1,5 & $(0,36-17,54)$ & 0,354 \\
\hline Beyblade & - & 0,9 & - & - & 0,3 & 2,3 & 7,52 & 0,104 \\
\hline Deymate & - & 0,9 & - & - & 0,3 & 2,3 & $(0,66-85,60)$ & 0,104 \\
\hline Oupimada & - & & - & - & & & 2,51 & \\
\hline Quemada & - & 0,4 & - & - & 0,3 & 0,1 & $(0,16-39,96)$ & 0,515 \\
\hline Instrumento musical & - & 0,8 & - & - & - & - & - & - \\
\hline
\end{tabular}

"Observação Direta. 'Web-CAAFE vs. Observação direta. "Web-CAAFE repetição das medidas.

Tabela 3. Efeito da exclusão de outliers sobre os valores médios de comportamentos sedentários e MET de atividades físicas, por método e repetição.

\begin{tabular}{|c|c|c|c|c|c|c|c|c|c|c|}
\hline \multirow{3}{*}{ Método } & \multirow{3}{*}{ Ocasião } & \multirow{3}{*}{$\mathrm{n}$} & \multicolumn{4}{|c|}{ Com outliers } & \multicolumn{4}{|c|}{ Sem outliers } \\
\hline & & & \multicolumn{2}{|c|}{$\mathrm{CS}^{*}$} & \multicolumn{2}{|c|}{ MET de $\mathrm{AF}^{\dagger}$} & \multicolumn{2}{|c|}{$\mathrm{CS}^{*}$} & \multicolumn{2}{|c|}{ MET de $\mathrm{AF}^{\dagger}$} \\
\hline & & & Média & IC 95\% & Média & IC $95 \%$ & Média & IC 95\% & Média & IC $95 \%$ \\
\hline \multirow{2}{*}{$\mathrm{OD}^{+}$} & 1 & 390 & 0,21 & $0,16-0,27$ & 4,3 & $3,94-4,67$ & 0,21 & $0,16-0,27$ & 4,3 & 3,94-467 \\
\hline & 2 & 94 & 0,32 & $0,19-0,45$ & 3,9 & $3,13-4,67$ & 0,32 & $0,19-0,45$ & 3,9 & $3,13-4,67$ \\
\hline \multirow{2}{*}{ Web-CAAFE } & 1 & 390 & 0,94 & $0,66-1,21$ & 7,6 & $5,36-9,78$ & 0,75 & $0,53-0,96$ & 4,8 & $3,86-5,63$ \\
\hline & 2 & 94 & 1,00 & $0,67-1,32$ & 7,4 & $5,30-9,56$ & 0,66 & $0,47-0,85$ & 5,1 & $4,14-6,10$ \\
\hline
\end{tabular}


Tabela 4. Validade e reprodutibilidade do Web-CAAFE através da regressão binomial negativa com efeitos fixos e randômicos.

\begin{tabular}{c|c|c|c|c|c|c}
\hline \multirow{2}{*}{ Efeitos } & \multicolumn{3}{|c|}{ CS $^{*}$} & \multicolumn{3}{c}{ MET de AF' } \\
\cline { 2 - 7 } & RI & Cl 95\% & P-valor & RI & Cl 95\% & P-valor \\
\hline Fixo & & & & & & \\
\hline Viés do Web-CAAFE & 2,9 & $2,05-4,10$ & 0,000 & 1,18 & $0,98-1,41$ & 0,079 \\
\hline Repetição & 1,15 & $0,86-1,53$ & 0,337 & 0,97 & $0,84-1,11$ & 0,642 \\
\hline Randômico & & & & & & \\
\hline Inter-sujeitos & 1,72 & $1,06-2,79$ & 0,000 & 1,1 & $0,87-1,40$ & 0,74 \\
\hline
\end{tabular}

"Total de comportamentos sedentários. ${ }^{\dagger}$ Atividades físicas.

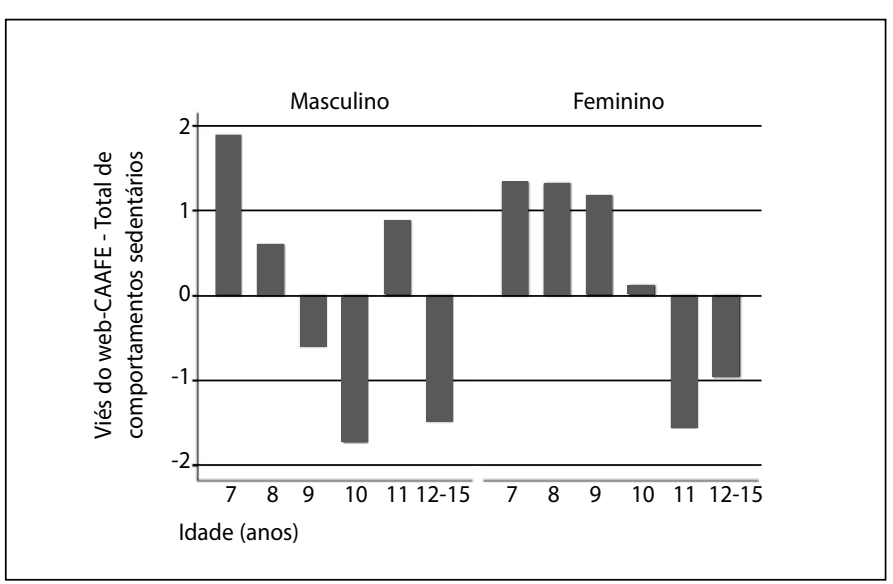

Figura 1. Vieses do Web-CAAFE em relação à observação direta, distribuídos por sexo e idade, para o total de comportamentos sedentários.

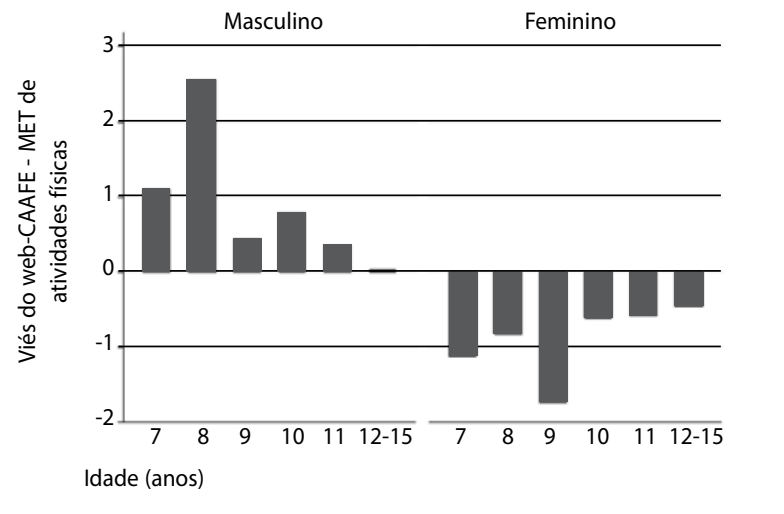

Figura 2. Vieses do Web-CAAFE em relação à observação direta, distribuídos por sexo e idade, para MET de atividades físicas.

Os resultados do presente estudo corroboram com a literatura com relação à reprodutibilidade do Web-CAAFE. Esses instrumentos ora superestimam ${ }^{23}$, ora subestimam ${ }^{24}$ a atividade física em comparação com acelerômetros, ou seja, os resultados ainda são conflitantes. Adicionalmente, esses estudos não avaliaram vieses em função do sexo ou da idade, possibilitando comparações mais adequadas com os resultados da presente pesquisa.

Em instrumentos impressos o autorrelato tende à superestimação da atividade física, mas, magnitude e distribuição desse viés não são tipicamente publicadas ${ }^{26}$. Há pouca informação também sobre vieses de autorrelatos computacionais, uma vez que a maioria dos estudos se limita ao cálculo de correlações ou concordância? .

O padrão de referência no atual estudo pode ter sido inadequado para validar os comportamentos sedentários, que na amostra pesquisada ocorriam predominantemente na sala de aula, enquanto as atividades físicas ocorriam essencialmente no recreio. Assim, a observação direta no recreio captou as atividades físicas, havendo pouco ou nenhum registro de comportamentos sedentários.

Ademais, enquanto o autorrelato no Web-CAAFE abrangeu um turno do dia (manhã ou tarde), o padrão de referência cobriu apenas um segmento do dia (recreio). Assim, os participantes provavelmente autorrelataram os comportamentos sedentários antes da aula (por exemplo, assistir TV durante o almoço / café da manhã antes de ir à escola) e durante a aula (sentados), levando também à superestimação.

Nota-se, que a escola não consentiu a observação durante a aula, para evitar interferências no trabalho do professor ou prejuízos na aprendizagem. Assim, estudos futuros devem adotar um padrão de referência que monitore os participantes durante todo o turno letivo, mas sem prejuízos para o processo pedagógico. Possibilitar o autorrelato das atividades do recreio em uma tela adicional no Web-CAAFE também pode incrementar a precisão na comparação com a observação direta. Ambas ações impactariam positivamente na avaliação da validade do instrumento.

Os vieses encontrados foram relacionados à idade e ao sexo dos participantes. Com relação à idade, os vieses foram maiores entre os estudantes mais jovens, podendo ser devidos a dificuldades na memória7,27 relacionadas ao padrão de atividade física, que é bastante variável e difícil de lembrar. Além disso, dificuldades de interpretação de questionários ${ }^{28}$ devido a características cognitivas e linguísticas próprias da faixa etária incluída no estudo também podem explicar esses vieses.

Com relação ao sexo dos participantes, os vieses podem ser devidos a diferenças no nível de envolvimento de meninos e meninas em atividades físicas. Uma hipótese seria de que meninos são frequentemente mais ativos fisicamente do que meninas ${ }^{29,30}$ e tendem a superestimar a atividade física, enquanto meninas tendem a subestimá-la por serem menos ativas.

Entre as limitações deste estudo apontam-se a amostra e o período de referência para a observação. A amostra de conveniência não permite generalizar os resultados, mas seu tamanho foi suficiente para validar diferenças entre os métodos comparados. Ademais, validade e confiabilidade avaliadas no estudo referem-se a um período do dia e não podem ser extrapoladas para o dia inteiro ou para contextos extracurriculares. Pesquisas futuras devem incluir escolas de tempo integral ou coletar dados do padrão de referência em contextos extracurriculares para melhorar a análise da validade.

Os pontos fortes abrangem a adoção da observação direta como critério de referência mais preciso do que o Web-CAAFE e independente da subjetividade dos participantes, a tentativa de minimizar as mudanças de comportamento resultante do padrão de referência, e a avaliação do viés da medida de autorrelato da atividade física.

Adicionalmente, a análise estatística permitiu avaliar simultaneamente reprodutibilidade e validade com alto poder de detecção da variabilidade do padrão de referência e de erros do tipo I e II, devido à inclusão de todas as informações disponíveis nos modelos de regressão, em vez de reduzir a amostra para os alunos que completaram as repetições das medidas de atividade física.

A exclusão dos outliers a partir dos critérios adotados também foi importante para dar mais precisão à análise. Outliers no Web-CAAFE ocorreram quando nenhuma (ausência de informação) ou muitas figuras foram selecionadas (excesso de informação), gerando respostas implausíveis. Assim, no refinamento do Web-CAAFE poder-se-ia considerar também a inclusão de um alerta para essas situações. 


\section{CONCLUSÃO}

A observação direta permitiu avaliar adequadamente validade e reprodutibilidade de MET de atividades físicas na amostra. Contudo, foi incapaz de captar precisamente os comportamentos sedentários, resultando em significativa superestimação. Os vieses relacionados à idade e ao sexo mostraram que o Web-CAAFE foi mais preciso quando aplicado à avaliação de MET de atividades físicas de estudantes mais velhos e que deve ser utilizado com cautela para comparar os meninos e as meninas na amostra estudada.

\section{AGRADECIMENTOS}

Os autores agradecem à Fundação de Amparo à Pesquisa do Estado da Bahia que financiou o estudo (Edital no 028/2012, Pedido no 1238/2013, Termo de Outorga no PES0049/2013).

Todos os autores declararam não haver qualquer potencial conflito de interesses referente a este artigo.

CONTRIBUIÇÕES DOS AUTORES: Cada autor contribuiu individual e significativamente para o desenvolvimento do manuscrito. Todos contribuíram de forma significativa na redação do manuscrito. GMJ (0000-0003-1702-217X)* concebeu o estudo e chefiou a coleta de dados em campo. MAAA (0000-0002-5383-3714)* concebeu o estudo e realizou a revisão do manuscrito. EK (0000-0001-6704-1673)* avaliou os dados da análise estatística e a revisão do manuscrito. LAD (0000-0003-461 1-9632)* coletou dados em campo, realizou a pesquisa bibliográfica e a revisão do manuscrito. Todos os autores contribuíram com o conceito intelectual do estudo. *Número ORCID (Open Researcher and Contributor ID).

\section{REFERÊNCIAS}

1. Ng M, Fleming T, Robinson M, Thomson B, Graetz N, Margono C, et al. Global, regional, and national prevalence of overweight and obesity in children and adults during 1980-2013: a systematic analysis for the Global Burden of Disease Study 2013. Lancet. 2014;384(9945):766-81.

2. Dodd CJ. Energy regulation in young people. J Sports Sci Med. 2007;6(3):327-36.

3. Laframboise MA, Degraauw C. The effects of aerobic physical activity on adiposity in school-aged children and youth: a systematic review of randomized controlled trials. J Can Chiropr Assoc. 2011;55(4):256-68.

4. Finelli C, Gioia S, La Sala N. Physical activity: an important adaptative mechanism for body-weight control. ISRN Obes. 2012;2012:675285.

5. Azevedo MR, Araújo CL, Cozzensa da Silva M, Hallal PC. Tracking of physicalactivity from adolescence to adulthood: a population-based study. Rev Saude Publica. 2007;41(1):69-75.

6. Pan American Heath Organization. Plan of action for the prevention of obesity in children and adolescents. Washington DC: PAHO; 2014. Disponivel em : http://www.paho.org/hq/index.php?option=com docman\&task=doc_view\&ltemid $=270 \&$ gid $=28890 \&$ lang $=p t$

7. Warren JM, Ekelund U, Besson H, Mezzani A, Geladas N, Vanhees L; Experts Panel. Assessment of physical activity - a review of methodologies with reference to epidemiological research: a report of the exercise physiology section of the, European Association of Cardiovascular Prevention and Rehabilitation. Eur J Cardiovasc Prev Rehabil. 2010;17(2):127-39.

8. Ekman A, Litton JE. New times, new needs; e-epidemiology. Eur J Epidemiol. 2007;22(5):285-92

9. Legnani E, Legnani RFS, Rech CR, Guimarães RF, Campos W. Instrumentos eletrônicos para avaliar atividade física em crianças: uma revisão sistemática. Motricidade. 2013;9(4):90-9.

10. Stumbo PJ, Weiss R, Newman JW, Pennington JA, Tucker KL, Wiesenfeld PL, et al. Web-enabled and improved software tools and data are needed to measure nutrient intakes and physical activity for personalized health research. J Nutr. 2010;140(12):2104-15.

11. da Costa FF, Schmoelz CP, Davies VF, Di Pietro PF, Kupek E, de Assis MA. Assessment of diet and physical activity of brazilian schoolchildren: usability testing of a web-based questionnaire. JMIR Res Protoc. 2013;2(2):e31.

12. Davies VF, KupekE, de Assis MA, Natal S, Di Pietro PF, Baranowski T.Validation of a web-based questionnaire to assess the dietary intake of Brazilian children aged 7-10 years. J Hum Nutr Diet. 2015;28(Suppl 1):93-102.

13. Costa FF. Desenvolvimento e avaliação de um questionário baseado na web para avaliar o consumo alimentar e a atividade física de escolares [tese de doutorado]. Florianópolis, SC: Universidade Federal de Santa Catarina; 2013.

14. Henderson KE, Grode GM, Middleton AE, Kenney EL, Falbe J, Schwartz MB. Validity of a measure to assess the child-care nutrition and physical activity environment. J Am Diet Assoc. 2011;111(9):1306-13
15. Ridley K, Ainsworth BE, Olds TS. Development of a compendium of energy expenditures for youth Int J Behav Nutr Phys Act. 2008;5:45.

16. Sirard JR, Pate RR. Physical activity assessment in children and adolescents. Sports Med. 2001;31(6):439-54

17. McMurray RG, Harrell JS, Bradley CB, Webb JP, Goodman EM. Comparison of a computerized physical activity recall with a triaxial motion sensor in middle-school youth. Med Sci Sports Exerc. 1998;30(8):1238-45

18. Ridley K, Dollman, J, Olds T. Development and validation of a Computer Delivered Physical Activity Questionnaire (CDPAQ) for children. Pediatr Exerc Sci. 2001;13:35-46.

19. Treuth MS, Sherwood NE, Butte NF, McClanahan B, Obarzanek E, Zhou A, et al. Validity and reliability of activity measures in African-American girls for GEMS. Med Sci Sports Exerc. 2003;35(3):532-9.

20. Lévesque L, Cargo M, Salsberg J. Development of the Physical Activity Interactive Recall (PAIR) for Aboriginal children. Int J Behav Nutr Phys Act. 2004;1 (1):8.

21. Ridley K, Olds TS, Hill A. The Multimedia Activity Recall for Children and Adolescents (MARCA): development and evaluation. Int J Behav Nutr Phys Act. 2006;3:10.

22. Philippaerts RM, Matton L, Wijndaele K, Balduck AL, De Bourdeaudhuij I, Lefevre J. Validity of a physica activity computer questionnaire in 12- to 18-year-old boys and girls. Int J Sports Med. 2006;27(2):131-6

23. Moore HJ, Ells LJ, McLure SA, Crooks S, Cumbor D, Summerbell CD, et al. The development and evalu ation of a novel computer program to assess previous-day dietary and physical activity behaviours in school children: the Synchronised Nutrition and Activity Program (SNAP). Br J Nutr. 2008;99(6):1266-74.

24. McLure SA, Reilly JJ, Crooks S, Summerbell CD. Development and evaluation of a novel computer -based tool for assessing physical activity levels in schoolchildren. Pediatr Exerc Sci. 2009;21(4):506-19.

25. Storey KE, McCargar L. Reliability and validity of Web-SPAN, a web-based method for assessing weight status, diet and physical activity in youth. J Hum Nutr Diet. 2012;25(1):59-68

26. Bates H. Daily physical activity for children and youth: a review and syntesis of the literature. Edmonton AL: Alberta Education; 2006.

27. 22: Corder K, Ekelund U, Steele RM, Wareham NJ, Brage S. Assessment of physical activity in youth. J Appl Physiol (1985). 2008;105(3):977-87.

28. Janz KF, Lutuchy EM, Wenthe P, Levy SM. Measuring activity in children and adolescents using self-report: PAQ-C and PAQ-A. Med Sci Sports Exerc. 2008;40(4):767-72.

29. Sallis JF, Prochaska JJ, Taylor WC. A review of correlates of physical activity of children and adolescents. Med Sci Sports Exerc. 2000;32(5):963-75.

30. Van Der Horst K, Paw MJ, Twisk JW, Van Mechelen W. A brief review on correlates of physical activity and sedentariness in youth. Med Sci Sports Exerc. 2007;39(8):1241-50 